\title{
Return to Sport After Shoulder Stabilization Procedures: A Criteria-Based Testing Continuum to Guide Rehabilitation and Inform Return-to-Play Decision Making
}

\author{
Thomas Otley, P.T., D.P.T., C.S.C.S., Heather Myers, P.T., D.P.T., S.C.S., L.A.T., A.T.C., \\ Brian C. Lau, M.D., and Dean C. Taylor, M.D.
}

\begin{abstract}
The athlete with shoulder instability poses a unique challenge to the sports medicine team. Clinical studies support surgical intervention followed by a phased approach to rehabilitation. In the latter phases, it is important to tailor this program to the individual's specific athletic needs, which requires ongoing qualitative assessment and objective measurement. Passing a return-to-sport testing battery has been shown to decrease the risk of recurrent instability. What is lacking in the literature is a consensus for how to best measure shoulder performance when the required athletic demands are widely varied by hand dominance, sport played, and playing position. Multiple upper-extremity tests have been described in the literature, but there is no consensus on which tests should be used to direct rehabilitation and to safely return the athlete to unrestricted athletic exposure. Using available evidence, we suggest a framework for returnto-play testing that integrates traditional rehabilitation phases with performance testing and graduated sports exposure. Level of Evidence: Level V, expert opinion.
\end{abstract}

$\mathbf{T}$ here is a high rate of return to sport after shoulder instability procedures, with most patients returning to the same level of play. Although many athletes are able to return to play, rates of recurrence can be significant. ${ }^{1}$ Multifactorial reasons for poor outcomes are described in the literature and can depend on the nature of the instability, choice of procedure, demands of the sport, and individual characteristics of the athlete. What is not described well in the literature is the condition (mobility, stability, performance, and so on) of

From Duke Sports Sciences Institute, Department of Physical Therapy and Occupational Therapy, Duke University Health System, Durham, North Carolina, U.S.A. (T.O., H.M.); and Duke Sports Sciences Institute, Department of Orthopedic Surgery, Duke University Health System, Durham, North Carolina, U.S.A. (B.C.L., D.C.T.).

The authors report no conflicts of interest in the authorship and publication of this article. Full ICMJE author disclosure forms are available for this article online, as supplementary material.

Received August 2, 2021; accepted September 15, 2021.

Address correspondence to Heather Myers, P.T., D.P.T., S.C.S., L.A.T., A.T.C., Duke Sports Sciences Institute-Physical Therapy, Box 3695, 3475 Erwin Rd,Durham, NC 27705, U.S.A.E-mail: heather.myers@duke.edu

(C) 2021 THE AUTHORS. Published by Elsevier Inc. on behalf of the Arthroscopy Association of North America. This is an open access article under the CC BY-NC-ND license (http://creativecommons.org/licenses/by-nc-nd/4.0/).

2666-061X/211107

https://doi.org/10.1016/j.asmr.2021.09.039 the shoulder at the time of clearance to return to play. Passing a return-to-sport testing battery has been shown to decrease the risk of recurrent instability. What is lacking in the literature is a consensus for how to best measure shoulder performance when the required athletic demands are widely varied by hand dominance, sport played, and playing position. Multiple upper-extremity tests have been described in the literature, but there is no consensus on which tests should be used to direct rehabilitation and to safely return the athlete to unrestricted athletic exposure. Our purpose was to describe a battery of objective measures designed to inform rehabilitation intervention and aid in returnto-sport decision making.

\section{Return to Sport After Stabilization Procedures}

The success of shoulder stabilization procedures, often described as a full return to the prior level of participation without recurrence, is influenced by many factors such as high-risk athletic demands, the amount of bone loss, the number of episodes of instability, and the direction of instability. A more appropriate measure of success may combine clinical outcomes with patient satisfaction, which has been directly linked to a 
patient's preoperative expectations. ${ }^{2}$ Specific to stabilization surgery for both primary and recurrent procedures, $92 \%$ of athletes expected to return to the same level of play and $79 \%$ expected instability to be completely resolved. It is interesting to note that the patients with the highest expectations were also those participating in the highest-risk athletic group (collision sports). ${ }^{2}$ For some athletes, perhaps because they graduate or choose to pursue a different sport after injury, a return to their prior sport is not a goal. Establishing goals and managing expectations as part of a shared decision-making process between the athlete and the provider should precede the initiation of interventions.

\section{Outcomes of Shoulder Stabilization Procedures}

In 2018, Ciccotti et al. ${ }^{3}$ performed a systematic review of return to sports participation in athletes undergoing procedures for anterior instability and found that most of the included studies-two-thirds-reported a rate of return to sports participation over $80 \%$. In a review of studies with at least 10 years' follow-up after primary arthroscopic Bankart procedures, the dislocation rate was $16 \%$; revision rate, $17 \%$; recurrent instability rate, $31.2 \%$; and instability arthropathy rate, 59.4\%. ${ }^{1}$ In athletes undergoing an open Bankart revision procedure, the commonly accepted recurrence rate is $3 \%$ to $5 \%$. This was consistent with the findings of a study of military cadets with a $3 \%$ recurrence rate and with only $4.5 \%$ reporting that they would decline surgery in hindsight. ${ }^{4}$ After the Latarjet procedure, $88 \%$ of athletes $(88.2 \%$ of collision and $90.3 \%$ of overhead athletes) return to sport, with $72.6 \%(69.5 \%$ of collision and $80.6 \%$ of overhead athletes) returning to the prior level of play. ${ }^{5}$ Most return at 5.8 months (range, 3.2-8 months). ${ }^{5}$ In a recent systematic review of the rate, timing, and criteria for return to play after posterior shoulder instability, Fried et al. ${ }^{6}$ reported that both collision and overhead athletes return to play at similar rates $(62.7 \%-100 \%)$ but overhead athletes are more likely to return at a lower level of play. Reinjury rates between $3.4 \%$ and $7 \%$ were reported. One retrospective investigation reported a lower likelihood of returning to sport after revision procedures. ${ }^{7}$

Ciccotti et al. $^{3}$ also extracted the criteria used for return-to-play decision making and found that no study included a scoring instrument. Time, strength, range of motion, absence of pain, radiographs, stability, and proprioception were included. However, time-most often 6 months-was the sole criterion in $75.8 \%$ of the studies. When range of motion, strength, and stability were included, the assessments were not described as objectively measured but rather as "full," "near normal," or "good static stability." 3 Hurley et al. performed a similar review of studies reporting on athletes undergoing the Latarjet procedure, which is promoted to provide a faster return to sport. The findings were similar: Time, imaging, clinical examination, strength, pain, and range of motion were listed as criteria, but none of the studies described objective means of assessment or predetermined thresholds to be met prior to release to unrestricted activity. After procedures addressing posterior instability, Fried et al. ${ }^{6}$ found no consensus for guidelines to determine when it is safe to return an athlete to play. Again, however, strength, range of motion, time (4-6 months with 6 months most commonly used), absence of pain, completion of a sport-specific rehabilitation protocol, and proprioception were reported. ${ }^{7}$

\section{Time and Clinical Examination Alone Are Not Adequate}

Although a period of 6 months is reported as the most frequently used criterion in return-to-sport decision making, it does not provide necessary information on the athlete's functional progress or readiness to return to athletic demands. A recent retrospective study emphasized the importance of using criteria-based return-to-sport testing (CBRST) in the decision-making process for returning athletes to sport after Bankart repair. ${ }^{8}$ The authors compared patients' return-to-play status based on the use of an established battery of tests. Athletes who did not undergo CBRST were 4.85 times more likely to experience recurrent instability after returning to sport and had a $22 \%$ rate of recurrence compared with a 5\% rate among athletes who were required to pass CBRST. ${ }^{8}$ Wilson et al. ${ }^{9}$ reported similar findings in a cohort of collegiate and high school athletes undergoing anterior or posterior stabilization surgery. At 6 months' follow-up, they found that only 5 of 43 patients passed the entire test battery. Only 7 of 43 passed all strength criteria. Alternatively, 26 of 43 passed both of the functional testing components. ${ }^{9}$ This finding establishes the potential for lingering strength deficits when time and functional testing are considered in isolation.

\section{Phased and Specific Rehabilitation Is Essential}

Not only do the aforementioned remaining deficits at 6 months underscore the importance of assessing physical and functional characteristics, they also reveal the need for high-quality rehabilitation to achieve the prior level of performance and to decrease recurrence in high-level sports. Progression from the early postoperative period to the rigorous demands of sports requires a strategic and informed approach. There is much agreement on rehabilitation guidelines including the 4 common phases (protective, restrictive, active, 
and function/sport) and, even more specifically, on the aims of early rehabilitation. Aside from grossly symmetrical strength and motion and the common time frame of 6 months, there are no well-implemented return-to-sport objective criteria for shoulder stabilization procedures. As mentioned previously, this lack of robust return-to-sport criteria may lead to an increased reinjury rate, but it also poses a challenge to determining the end goals for rehabilitation. When determining the rehabilitation trajectory, it is important to start with the end in mind. This begins with establishing the athlete's expectations and goals through the shared decision-making process. Without a clear approach and objective criteria, one is unable to accurately determine readiness for return to sporting demands and to subsequently reduce the risk of reinjury.

In a 2016 consensus statement from the World Congress in Sports Physical Therapy, Ardern et al. ${ }^{10}$ described a 3-step return-to-sport progression to guide end-stage decision making. This approach has been referred to as the " 3 P Program: Performance, Practice, and Play" and has been mentioned in previous clinical commentaries on return to play after shoulder injury but not specifically to shoulder instability. ${ }^{11}$ After the resumption of daily activities and the return of foundational strength and range of motion, the athlete enters the first phase: the performance phrase. The performance phase is focused on the restoration of function through sport-specific training for athletes returning to competition. Second, the practice phase follows the gradual progression of training participation via a titrated increase in the time, intensity, and number of repetitions performed by the athlete during practice. Finally, after the successful completion of a thorough controlled practice progression, the athlete is evaluated for readiness to resume competition in game situations at full effort. ${ }^{10}$

Rehabilitation plays a significant role in guiding midto late-stage progression and should include a stepwise progression for exposing athletes to the specific demands of their sport. Guidelines such as the 3 P Program provide an excellent framework for conceptualizing a graduated return to participation and play, but they leave an opportunity to be complemented with objective criteria when making returnto-play decisions. For example, one is unlikely to adequately address deficits in strength if such deficits are unmeasured. The use of criteria-based testing provides valuable information that can inform the rehabilitation plan along the full continuum of recovery while also setting benchmarks for physical performance at the point of return to sports participation.

\section{Elements of Comprehensive Test Battery}

The primary goal of a test battery is to prevent recurrence or the development of secondary sequelae by setting benchmarks to inform providers and athletes, as well as their stakeholders. With this in mind, a test battery should include elements of what is required for sports participation and what is required for athletes to protect themselves during live play. We propose that these elements should include assessments of psychological readiness and absence of kinesiophobia; adequate sport-specific range of motion; the ability to both develop and resist force; and scapulohumeral stability and endurance. Means of testing these criteria are discussed in the following sections and are summarized in Table 1.

\section{Psychological Readiness}

A number of patient-reported outcome measures (PROMs) include components to identify patients who will have psychological difficulties returning to play. Athletes with more positive psychological responses, such as motivation, confidence, and a low level of fear, are more likely to return to sport faster and to return their preinjury level of participation; therefore, elements of psychological readiness should be taken into account. ${ }^{12}$ Examples of these PROMs are the Western Ontario Shoulder Instability Index (WOSI), the Shoulder Instability Return to Sport Index (SIRSI), and the Kerlan-Jobe Orthopedic Clinic Shoulder and Elbow Score (KJOC). The WOSI, 1 of the 4 most used PROMs in shoulder instability, includes domains of physical symptoms; sport, recreation, and work activities; lifestyle; and emotion. Whittle et al. ${ }^{13}$ confirmed the appropriate reliability, validity, and responsiveness of the WOSI in the setting of shoulder instability with an endorsement of additional outcomes. Wilk et al. ${ }^{11}$ suggested a score greater than 90 for return to practice and a score greater than 95 for return to full competition for athletes returning to play after the treatment of all shoulder conditions. Lacking from the WOSI is the ability to predict future pain and disability. One PROM, the KJOC, has been investigated for its predictive ability specific to both upper-extremity conditions and sports participation. The KJOC is widely used in participants in overarm throwing sports, such as baseball, but has also been reported in participants in softball, swimming, and elite canoe. ${ }^{14}$ Symptomatic athletes in several different sports scored lower on the KJOC than those who were asymptomatic or those who reported a history of pain. The value of including the KJOC in a CBRST battery is its predictive ability to detect increased injury risk, which is currently accepted as a score lower than 90 for participants in baseball, with a score lower than 88 for participants in other upper extremity-dominant sports, such as canoe/ kayak, reporting similar metrics. ${ }^{14}$ With respect to specifically quantifying psychological readiness to return to sport, the developers of the Shoulder Instability Return to Sport Index (SIRSI) aimed to model a 
PROM

WOSI

SIRSI

KJOC

\section{Performance measure}

Isometric strength of ER and IR at

$0^{\circ}$ and $90^{\circ}$ using instrumented

(handheld or fixed)

dynamometry
The WOSI includes 21 questions across 4 domains. Each Physical symptoms question is scaled from 0 (best) to 100 (worst). The original score was reported as the total sum of all questions. ${ }^{13}$ For better comparisons across respondents, a modified score was created: Modified score $=100-$ (Original score/21)

The SIRSI includes 12 questions with an 11-point Likert scale $(0-10)$. The total score is equal to the sum of the 12 values divided by 120 and expressed as a percentage. ${ }^{1}$ The KJOC includes 10 questions, each answered on a 10 $\mathrm{cm}$ visual analog scale, and measured to the nearest millimeter. Scores are added and expressed as a percentage with a range of $0 \%$ (worst) to $100 \%$ (best). ${ }^{14}$ For competitive overhead athletes, players without pain scored $94.4 \%$, players with pain scored $61.0 \%$, and players not able to play scored $44.3 \%{ }^{28}$

Shoulder IR and ER (at $0^{\circ}$ and $90^{\circ}$ of abduction) are measured for an isometric hold of 5 seconds for at least 2 trials on both sides. The trials are averaged and compared bilaterally. Measures can also be normalized to body weight.

The athlete lies prone with the arm off the edge of the table and then moves the arm to $90^{\circ}$ of horizontal abduction while holding a weight equal to $2 \%$ of body weight (rounded to the nearest half pound). The arm raises are repeated until position and technique criteria fail. The score is reported as the number of completed repetitions and is compared bilaterally. ${ }^{23}$ In the modified version, the abduction position is held as an isometric contraction to failure.

The ASH uses closed-chain assessment using fixed force plates to provide a platform for measuring the rate of force development and peak torque in the I, Y, and positions. ${ }^{18}$

activities

Lifestyle

Emotion

Psychological readiness

\section{Symptoms (pain)}

Rotator cuff strength musculature

Sport, recreation, and work

Upper-extremity performance

Normative references for both IR and ER strength and IR/ER strength ratios are available for some sport- and age-matched comparisons. In general, athletes should aim for a limb symmetry index within $10 \%$ for bilateral comparisons of IR and ER strength. In throwers, this should be $>100 \%$ if the affected side is the throwing shoulder. IR/ ER ratios in healthy athletes range from 0.65 to 0.99 depending on position, sex, and sport. For throwers, 0.72-0.76 would be an appropriate goal for the IR/ER ratio ${ }^{1}$

Endurance of posterior shoulder

imb symmetry index within $10 \%$

Modified version: 46 seconds for female individuals and 47 seconds for male individuals ${ }^{24}$

Peak force and rate-of-force development
Limb symmetry index within 10\%

The minimum detectable change was between 13.2 and $25.9 \mathrm{~N}^{18}$ 
Test or Outcome Measure

UQ-YBT

CKCUEST

OAHT

SSASP against a wall and is instructed to press a $2-\mathrm{kg}$ medicine ball for the maximum horizontal distance. This distance is compared bilaterally. ${ }^{25}$ closed-chain assessment. The athlete stabilizes himself or herself in a plank position on $1 \mathrm{arm}$ while reaching as far as possible in 3 different directions with the opposite upper extremity. Reach distances are normalized to arm length and compared bilaterally with a goal of symmetry. ${ }^{21}$

The athlete assumes a plank position with the hands 36 inches apart and then reaches across the body to tap the other hand, alternating touches for as many repetition as possible for 15 seconds. The mean of three 15 -second trials is reported. For female athletes, the plank position is performed on the knees ${ }^{19}$ To normalize to body size, young athletes or small-statured athletes may modify the position by placing the hands under the shoulder rather than keeping them 36 inches apart. ${ }^{2}$

The OAHT is a high-level, closed-chain assessment. The athlete assumes a 1-arm push-up position and then uses that arm to hop on and off a $10.2-\mathrm{cm}$ step 5 times as quickly as possible. Time is compared bilaterally.
The athlete assumes a long-sitting position with the trunk

Construct

Criteria

The UQ-YBT allows upper-extremity and trunk stability

\section{Trunk stability}

Upper-extremity stability

Speed

Power

Impact

Tolerance

Strength

Power

Expected asymmetry of 3\%-13\% between

dominant and nondominant arms

Expected asymmetry of 4.4 seconds between dominant and nondominant sides ${ }^{22}$

(touches is considered normal and reflective of a decreased injury risk. ${ }^{19}$ Normative values specific to sex, age, and sport are available in the literature. 


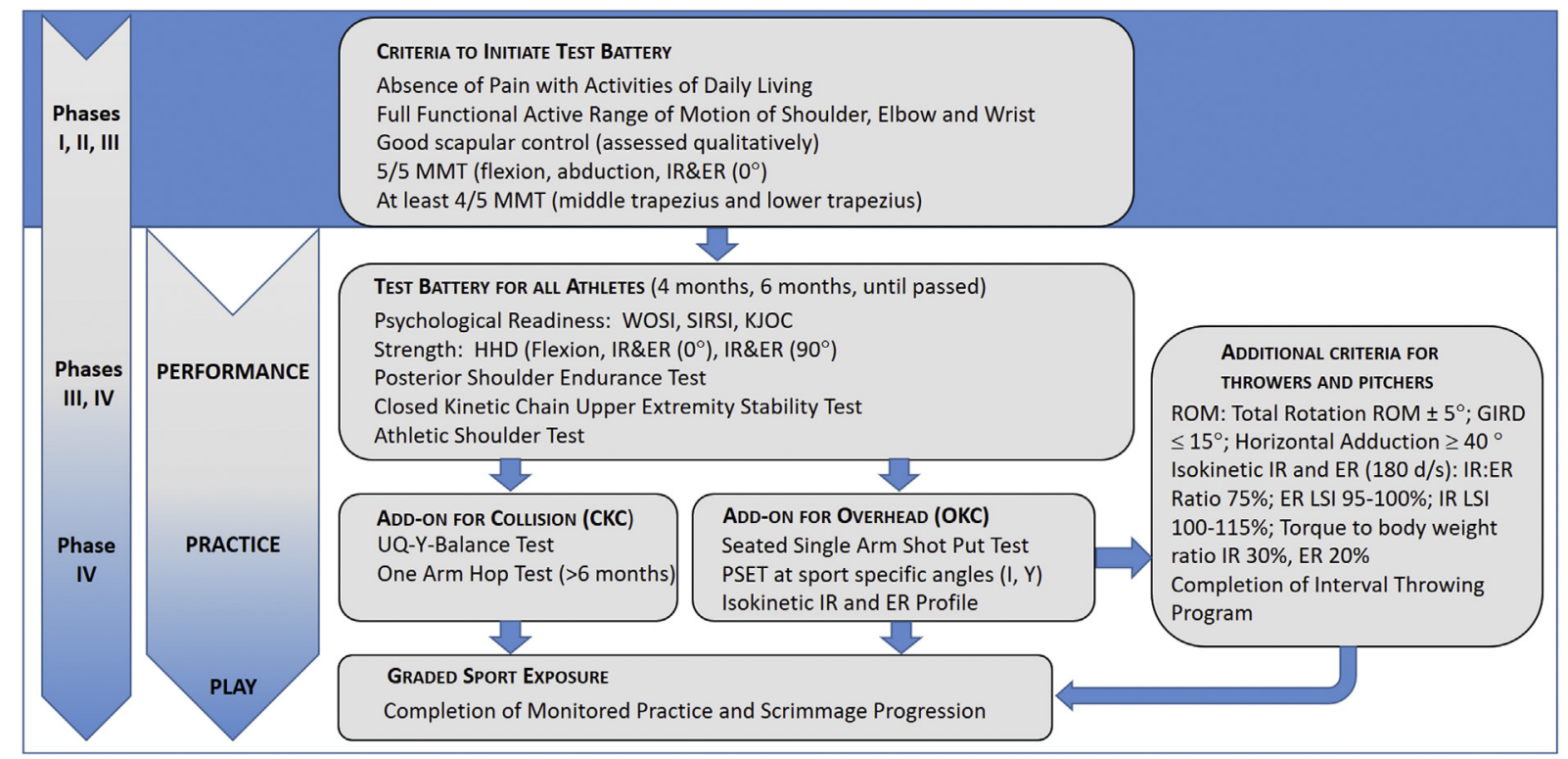

Fig 1. Framework for sequentially testing athletes recovering from shoulder stabilization procedures. This stepwise structure suggests criteria to be administered as the athlete progresses through phased rehabilitation and graduated exposure toward full athletic participation. (CKC, closed kinetic chain; d/s, degrees per second; ER, external rotation; GIRD, glenohumeral internal rotation deficit; HHD, handheld dynamometry; IR, internal rotation; KJOC, Kerlan-Job Orthopedic Clinic Shoulder and Elbow Score; LSI, limb symmetry index [involved/uninvolved × 100]; MMT, manual muscle testing; OKC, open kinetic chain; PSET, Posterior Shoulder Endurance Test; ROM, range of motion; SIRSI, Shoulder Instability Readiness to Return to Sport Index; UQ, Upper Quarter; WOSI, Western Ontario Shoulder Instability Index.)

validated PROM prevalent in the anterior cruciate ligament literature and apply it to athletes who are ready to return to sport after an episode of shoulder instability, whether treated surgically or nonoperatively. The developers reported significant difference in scores, which were worse in players who had not returned to the same sport after an episode of instability. ${ }^{15}$

\section{Sport-Specific Range of Motion}

The nature of instability procedures, along with a period of immobilization during the protective phase of rehabilitation, requires a gradual restoration of normal and symmetrical range of motion. The full and painfree active range of motion required for nonathletic activities of daily living should be achieved prior to undergoing a return-to-sport test battery so that testing positions can be assumed without discomfort or stress to soft tissues. Some athletes require a range of motion beyond what is considered the normal limits as part of the biomechanical adaptation to the demands of the sport. Sports such as swimming and gymnastics reward bilateral symmetrical hypermobility, whereas other sports, such as baseball, volleyball, and racquet sports, require specific asymmetrical adaptations. When including range of motion as a criterion for return to sport, the clinician must consider these additional requirements because failing to do so may result in poor mechanics with overcompensation at the distal or proximal joint. Although some adaptive range-ofmotion metrics are well documented, such as those observed in baseball pitchers, others are less established. A robust set of reference values based on sport, competition level, sex, and age or maturity is lacking in the literature. We suggest using the criteria in Figure 1 and Table 1 for baseball players ${ }^{11}$ and endorse further literature review for established norms that may apply to other specific populations.

\section{Strength and Force Development}

The ability to generate, sustain, and/or attenuate force across the shoulder joint is a requirement of athletic performance and should be assessed routinely throughout rehabilitation. In early phases, the clinician may choose to perform manual muscle testing to objectively grade performance and symmetry in all planes. As the athlete approaches symmetry, handheld dynamometry may be implemented to provide a more objective and reliable comparison and may expose deficits that are too subtle to be appreciated with manual testing. We suggest a manual muscle testing score of 5 of 5 for shoulder flexion, abduction, internal rotation (IR) (neutral), and external rotation (ER) 

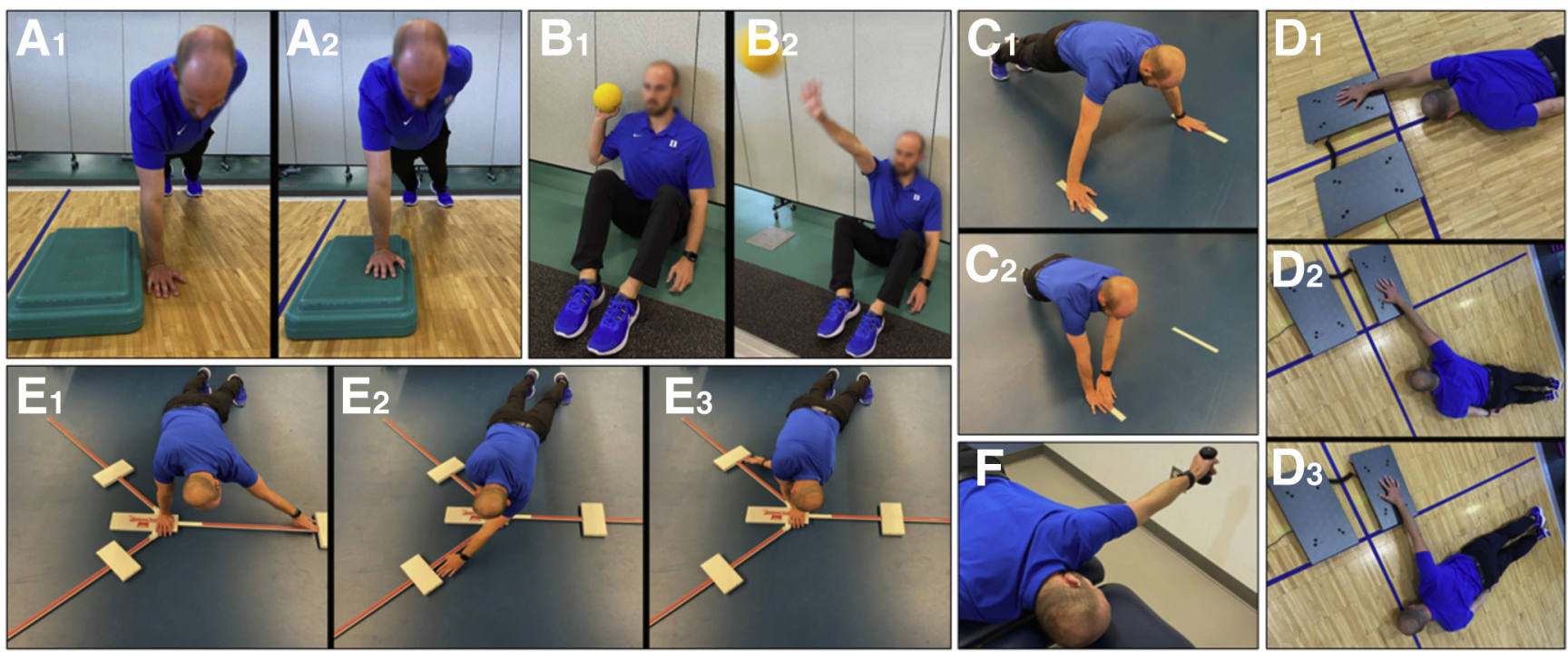

Fig 2. After shoulder stabilization procedures, athletes should undergo a battery of performance tests determined by the demands of their particular sport. This may include the following open- and closed-chain performance tests for strength, power, speed, endurance, and stability. (A) In the One-Arm Hop Test (OAHT), the athlete begins in a 1-arm plank position (1) and hops on (2) and off (1) a 10.2-cm step 5 times for speed. (B) The athlete assumes the start position (1) of the Seated Single Arm Shot Put (SSASP) and presses (2) the 2-kg ball as far as possible. (C) During the Closed Kinetic Chain Upper Extremity Stability Test (CKCUEST), the athlete begins in a plank position (1) with the hands 36 inches apart and then alternates touches between tapes

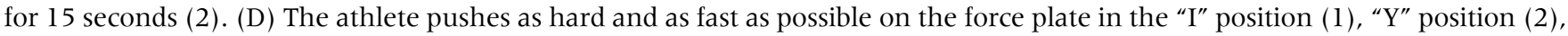
and " $\mathrm{T}$ " position (3). (E) For the Y-Balance Test of the Upper Quarter (UQ-YBT), the athlete maintains a plank position and reaches as far as possible in the medial (1), superolateral (2), and inferolateral (3) directions. (F) The athlete maintains a weighted, horizontally abducted arm during the Posterior Shoulder Endurance Test (PSET).

(neutral) and a score of at least 4 of 5 for the middle and lower trapezius prior to initiation of advanced test battery items. Handheld dynamometry may prove most useful for shoulder IR and ER (neutral) and for IR and ER at $90^{\circ}$ of abduction because there are normative references for strength and IR/ER strength ratios available in the literature. ${ }^{16,17}$

Although less widely available, isokinetic dynamometry may reveal additional asymmetries not captured with isometric resistance. The limb symmetry index for IR and ER peak torque, as well as IR/ER peak torque ratios, can be indicative of further strength deficits. Achieving adequate peak force is a necessary benchmark for the return to athletic activities; however, in the lower-extremity literature, deficits in the rate of force development have been present even when peak force is symmetrical. In the upper extremity, both protection of the joint and explosive performance require a fast rate of force development. Fixed force plates provide a platform for measuring the rate of force development along with peak torque. Ashworth et al. ${ }^{18}$ have proposed a test of force production and peak force assessment across a long lever arm (I, Y, and T positions) similar to functional athletic positions such as tackling and throwing. The Athletic Shoulder (ASH) Test may provide further insight into one's ability to perform safely and effectively in the athletic setting (Fig 2).

\section{Scapulohumeral Stability and Endurance}

The ability of an athlete to dynamically stabilize the shoulder complex during high-velocity and highimpact play is paramount to prevention of both acute instability recurrence and chronic symptomatic conditions that may develop in its absence. Athletes may use the upper extremity in either open or closed kinetic chain positions, and testing should reflect the demands of the sport. Several open- and closed-chain tests that require dynamic and static stability have been described in the literature. Examples of tests frequently reported for this population are the Closed Kinetic Chain Upper Extremity Stability Test (CKCUEST), Posterior Shoulder Endurance Test (PSET), Seated Single Arm Shot Put Test (SSASP), One-Arm Hop Test (OAHT), and Y-Balance Test of the Upper Quarter (UQ-YBT) (Fig 2). The CKCUEST requires the athlete to assume a plank position with the hands 36 inches apart and then reach across the body to tap the other hand. The athlete alternates touches for as many repetitions as possible for 15 seconds. The mean of three 15 -second trials is reported. For female athletes, the plank position is performed on the knees. ${ }^{19}$ To normalize to body size, Hollstadt et al. ${ }^{20}$ described a further modification for 
athletes who require a shorter reach distance (i.e., young athletes or small-statured athletes) by placing the hands under the shoulder rather than keeping them 36 inches apart. This is a reliable and valid test that places high demand on the scapular stabilizers and involves the full upper extremity and the trunk. ${ }^{20}$ Normative values have been reported among multiple sports, between sexes, and among ages, with a generally accepted score of 21 touches or greater as normal and reflective of decreased injury risk.

Similarly to the CKCUEST, the UQ-YBT incorporates full upper-extremity and trunk stability in a closedchain position on 1 arm while the athlete reaches as far as possible in 3 different directions with the opposite arm. Reach distances are normalized to arm length and compared bilaterally with a goal of symmetry. ${ }^{21}$ Further comparison to normative data for age, sex, sport, and competition level may be available in the literature.

The OAHT is another, although higher-level, closedchain assessment. The athlete is asked to assume a 1 -arm push-up position and then uses that arm to hop on and off a 10.2-cm step 5 times as quickly as possible. Time is compared bilaterally, with a 4.4-second difference indicating the expected asymmetry between the dominant and nondominant arms. ${ }^{22}$

The PSET was originally described as an isotonic endurance test for the shoulder stabilizers. The athlete lies prone with the arm off the edge of the table and then moves the arm to $90^{\circ}$ of horizontal abduction while holding a weight equal to $2 \%$ of body weight (rounded to the nearest half pound). ${ }^{14,23}$ The arm raises are repeated until position and technique criteria fail. The score is reported as the number of completed repetitions and is compared bilaterally, with a suggested goal of a limb symmetry index within $10 \%$. A modified version of the PSET has been described as an isometric hold for time, rather than isotonic repetitions, and this may be more appropriate and efficient for higher-level, stronger athletes. Evans et al. ${ }^{24}$ suggested a cutoff of 46 seconds for female individuals and 47 seconds for male individuals because less time was associated with shoulder pain.

The SSASP is designed to measure multiple-joint strength and power of the upper extremity in an openchain position. Athletes assume a long-sitting position with the trunk against a wall. They are instructed to press a $2-\mathrm{kg}$ medicine ball for the maximum horizontal distance. This distance is compared bilaterally, with $3 \%$ to $13 \%$ of expected asymmetry between the dominant and nondominant arms. ${ }^{25}$

\section{Challenges Unique to Upper-Extremity Assessment}

The challenge in establishing a consensus on a singular battery for upper-extremity return-to-sport testing lies in selecting tests and measures that effectively assess differing sport-specific demands. The closed versus open kinetic chain testing position is one of the prime examples of this debate. Whereas many traditional return-to-sport tests, such as the CKCUEST, OAHT, and UQ-YBT, use a closed-chain approach to assess shoulder stability and function, many sports require open-chain activity almost exclusively.

When considering either open- or closed-chain functional testing, the mechanism of injury should be considered. A systematic review by Sheehan et al. ${ }^{26}$ suggests that most traumatic dislocations are due to a fall on an outstretched hand-type mechanism and that most anterior dislocations occur with a sudden loading force in various degrees of shoulder abduction, ER, and extension. In contrast, posterior shoulder instability often presents with an insidious onset rather than after an acute incident. Posterior instability is commonly seen in athletes with sporting demands of increased posterior loading, such as American football linemen and weight lifters, as well as participants in sports requiring increased shoulder range of motion, such as swimming and gymnastics. ${ }^{6}$ Assessing the stability of motion related to the mechanism of injury should be one factor considered in determining the type of test to be administered.

Additionally, the athlete's sport-specific demands can provide a valuable context for which testing elements to include. As previously mentioned, test batteries should include elements of what is required for sports participation and for athletes to protect themselves during live play. Thus, contact or collision sports should involve some assessment of closed-chain stabilization and control such as the OAHT or Athletic Shoulder (ASH) Test. Overhead and throwing athletes should be tested in the open-chain position with tests such as the SSASP and PSET, as well as measurement of ER and IR strength in the upper ranges of elevation.

\section{Proposed Testing and Return-to-Participation Framework}

On the basis of the previous recommendations for return-to-sport testing combined with traditional rehabilitation phases and the 3 P Program, we propose the following stepwise progression of criteria-based testing for return to play after shoulder stabilization procedures. Figure 1 illustrates the interaction of these 3 elements along the rehabilitation continuum. Phases I, II, and III coincide with the return to full functional range of motion, absence of pain, and good scapular and shoulder strength. After meeting these criteria, the athlete may progress to performance-based strengthening and preparation for sport demands. In phases III and IV, the athlete undergoes a period of focused strength training and introductory upper-extremity stability work. Testing at this phase assesses psychological readiness, dynamometry strength measures, 
periscapular endurance, and closed-chain stability and strength. These tests are introduced between months 4 and 6 based on the athlete's individual progress. When the athlete passes this initial battery, he or she begins to practice with limited exposure. There are additional testing considerations for collision, overhead, and throwing athletes, which may be included as add-on criteria for a more specific assessment tailored to athletic demands. Finally, in phase IV, the athlete completes a graded exposure to a practice and scrimmage progression and passes all criteria-based testing prior to returning to unrestricted competition.

Owing to the limited availability of literature on return-to-sport testing after shoulder instability procedures, future studies should focus on validation of testing batteries. Because the proposed return-to-play testing framework is theoretical in nature, readers should consider their settings and patient populations when integrating these recommendations.

\section{Limitations}

There are many benefits of using CBRST. However, even batteries using valid and reliable tests are not without limitations. When compared with return-tosport batteries for anterior cruciate ligament and other lower-extremity injuries, far fewer studies have examined upper-extremity reinjury rates, most of which had small sample sizes. There is also limited available evidence on the predictability of the reinjury rate based on passing upper-extremity return-to-sport criteria.

\section{Conclusions}

The return to sport after shoulder stabilization procedures is generally successful, although reinjury and other symptomatic sequelae do occur. Shared returnto-sport decision making is vital to the health and well-being of the athlete. Informing these decisions with objective criteria through return-to-sport testing is an effective way to ensure readiness for return to sporting demands and to reduce the risk of reinjury. There is limited reporting of criteria-based decision making being used in contemporary practice, with most criteria for the return to sports based on time alone. We propose a framework of objective measures designed to inform interventions across the continuum of recovery and to aid in making decisions relating to a patient's participation goals. The framework includes elements of traditional rehabilitation phases, objective testing to better address the identified deficits and to determine readiness for progression, and guidance on integration into practice and competition. Return-to-sport testing should play a complementary role to inform decision making in this return-to-play model. The only activity that fully tests the demands of a sport is the sport itself. Performance criteria should not be used solely as a clearance examination; rather, they should be used to determine readiness to participate in graduated sport exposure.

\section{References}

1. Murphy AI, Hurley ET, Hurley DJ, Pauzenberger L, Mullett H. Long-term outcomes of the arthroscopic Bankart repair: A systematic review of studies at 10-year follow-up. J Shoulder Elbow Surg 2019;28:2084-2089.

2. Plath JE, Saier T, Feucht MJ, et al. Patients' expectations of shoulder instability repair. Knee Surg Sports Traumatol Arthrosc 2018;26:15-23.

3. Ciccotti MC, Syed U, Hoffman R, Abboud JA, Ciccotti MG, Freedman KB. Return to play criteria following surgical stabilization for traumatic anterior shoulder instability: A systematic review. Arthroscopy 2018;34:903-913.

4. Uhorchak JM, Arciero RA, Huggard D, Taylor DC. Recurrent shoulder instability after open reconstruction in athletes involved in collision and contact sports. Am J Sports Med 2000;28:794-799.

5. Hurley ET, Montgomery C, Jamal MS, et al. Return to play after the Latarjet procedure for anterior shoulder instability: A systematic review. Am J Sports Med 2019;47: 3002-3008.

6. Fried JW, Hurley ET, Duenes ML, et al. Return to play after arthroscopic stabilization for posterior shoulder instability-A systematic review. Arthrosc Sports Med Rehabil 2021;3:e249-e256.

7. Vaswani R, Arner J, Freiman H, Bradley JP. Risk factors for revision posterior shoulder stabilization in throwing athletes. Orthop J Sports Med 2020;8:2325967120967652.

8. Drummond Junior M, Popchak A, Wilson K, Kane G, Lin A. Criteria-based return-to-sport testing is associated with lower recurrence rates following arthroscopic Bankart repair. J Shoulder Elbow Surg 2021;30:S14-S20.

9. Wilson KW, Popchak A, Li RT, Kane G, Lin A. Return to sport testing at 6 months after arthroscopic shoulder stabilization reveals residual strength and functional deficits. J Shoulder Elbow Surg 2020;29:S107-S114.

10. Ardern CL, Glasgow P, Schneiders A, et al. 2016 Consensus statement on return to sport from the First World Congress in Sports Physical Therapy, Bern. $\mathrm{Br} J$ Sports Med 2016;50:853-684.

11. Wilk KE, Bagwell MS, Davies GJ, Arrigo CA. Return to sport participation criteria following shoulder injury: A clinical commentary. Int J Sports Phys Ther 2020;15: 624-642.

12. Ardern CL, Taylor NF, Feller JA, Webster KE. A systematic review of the psychological factors associated with returning to sport following injury. Br J Sports Med 2013;47:1120-1126.

13. Whittle JH, Peters SE, Manzanero S, Duke PF. A systematic review of patient-reported outcome measures used in shoulder instability research. J Shoulder Elbow Surg 2020;29:381-391.

14. Powell A, Williamson S, McCaig S, Heneghan NR, Horsley I. An investigation of a Kerlan-Jobe Orthopaedic Clinic shoulder and elbow score in elite canoe slalom: Establishing measurement properties to make practice recommendations. Phys Ther Sport 2021;50:15-21. 
15. Gerometta A, Klouche S, Herman S, Lefevre N, Bohu Y. The Shoulder Instability-Return to Sport after Injury (SIRSI): A valid and reproducible scale to quantify psychological readiness to return to sport after traumatic shoulder instability. Knee Surg Sports Traumatol Arthrosc 2017;26:1-9.

16. Cools AMJ, Vanderstukken F, Vereecken F, et al. Eccentric and isometric shoulder rotator cuff strength testing using a hand-held dynamometer: Reference values for overhead athletes. Knee Surg Sports Traumatol Arthrosc 2016;24:3838-3847.

17. Riemann BL, Davies GJ, Ludwig L, Gardenhour H. Handheld dynamometer testing of the internal and external rotator musculature based on selected positions to establish normative data and unilateral ratios. J Shoulder Elbow Surg 2010;19:1175-1183.

18. Ashworth B, Hogben P, Singh N, Tulloch L, Cohen DD. The Athletic Shoulder (ASH) test: Reliability of a novel upper body isometric strength test in elite rugby players. BMJ Open Sport Exerc Med 2018;4:e000365.

19. Goldbeck TG, Davies GJ. Test-retest reliability of the closed kinetic chain upper extremity stability test: A clinical field test. J Sport Rehabil 2000;9:35-45.

20. Hollstadt K, Boland M, Mulligan I. Test-retest reliability of the Closed Kinetic Chain Upper Extremity Stability Test (CKCUEST) in a modified test position in Division I collegiate basketball players. Int J Sports Phys Ther 2020;15: 203-209.

21. Gorman PP, Butler RJ, Plisky PJ, Kiesel KB. Upper Quarter Y Balance Test: Reliability and performance comparison between genders in active adults. J Strength Cond Res 2012;26:3043-3048.

22. Falsone SA, Gross MT, Guskiewicz KM, Schneider RA. One-arm hop test: Reliability and effects of arm dominance. J Orthop Sports Phys Ther 2002;32:98-103.

23. Moore SD, Uhl TL, Kibler WB. Improvements in shoulder endurance following a baseball-specific strengthening program in high school baseball players. Sports Health 2013;5:233-238.

24. Evans NA, Konz S, Nitz A, Uhl TL. Reproducibility and discriminant validity of the Posterior Shoulder Endurance Test in healthy and painful populations. Phys Ther Sport 2021;47:66-71.

25. Chmielewski TL, Martin C, Lentz TA, et al. Normalization considerations for using the unilateral seated shot put test in rehabilitation. J Orthop Sports Phys Ther 2014;44:518-524.

26. Sheehan SE, Gaviola G, Gordon R, Sacks A, Shi LL, Smith SE. Traumatic shoulder injuries: A force mechanism analysis-glenohumeral dislocation and instability. AJR Am J Roentgenol 2013;201:378-393.

27. Su F, Allahabadi S, Bongbong DN, Feeley BT, Lansdown DA. Minimal clinically important difference, substantial clinical benefit, and patient acceptable symptom state of outcome measures relating to shoulder pathology and surgery: A systematic review. Curr Rev Musculoskelet Med 2021;14:27-46.

28. Alberta FG, ElAttrache NS, Bissell S, et al. The development and validation of a functional assessment tool for the upper extremity in the overhead athlete. Am J Sports Med 2010;38:903-911. 